\title{
THE DERANGEMENT PROBLEM RELATIVE TO THE MAHONIAN PROCESS
}

\section{JESSICA DELFERT, HILLARY EINZIGER, and DON RAWLINGS}

\author{
Received 23 September 2002
}

\begin{abstract}
We survey $n$ ! plus $q$-derangement problems. Solutions to four Mahonian statistics arising in connection with cycle placement rules are presented. A few conjectures are also made.
\end{abstract}

2000 Mathematics Subject Classification: 60K99, 05A05, 05A30.

1. Introduction. Let $S_{n}$ denote the symmetric group on $\{1,2, \ldots, n\}$. A permutation $\sigma \in S_{n}$ is said to be a derangement of $\{1,2, \ldots, n\}$ if $\sigma(i) \neq i$ for $1 \leq i \leq n$. We let $D_{n}$ be the set of derangements of $\{1,2, \ldots, n\}$ and set $d_{n}=$ $\left|D_{n}\right|$. A classic problem of combinatorics is to compute the probability that a permutation selected at random is a derangement. Typically, the principle of inclusion-exclusion is used to prove that

$$
d_{n}=n ! \sum_{k=0}^{n} \frac{(-1)^{k}}{k !}
$$

The exact solution of the derangement problem is then given by $d_{n} / n$ !. Furthermore, (1.1) immediately implies the celebrated fact that

$$
\lim _{n \rightarrow \infty} \frac{d_{n}}{n !}=\frac{1}{e}
$$

Historically, $d_{n} / n$ ! was first computed in 1708 by de Montmort [4] in the more general context of the problème des rencontres (the matching problem). Since de Montmort's day, a seemingly endless stream of authors have considered variations on the derangement problem. We are not different. We survey a plethora of more than $n ! q$-derangement problems relative to the Mahonian process. Besides a brief discussion of the $q$-derangement problems solved by Garsia and Remmel [5], Gessel [7], Griffin [8], and Wachs [16], we present solutions relative to four Mahonian statistics. Although two of the four distributions we consider are not new, our proofs are.

2. A thumbnail sketch of the $q$-calculus. The $q$-calculus is a vast subject. Gasper and Rahman [6] provide a comprehensive account. Only the material we need is touched upon here. 
An object $X(q)$ is said to be a $q$-analog of $X$ if $\lim _{q \rightarrow 1} X(q)=X$. The standard $q$-analogs of an integer $n \geq 0$ and its factorial, respectively, are

$$
[n]=1+q+q^{2}+\cdots+q^{n-1}, \quad[n] !=[1][2] \cdots[n],
$$

where the empty sum is 0 and the empty product is 1 . As a partial sum of a geometric series, $[n]=\left(1-q^{n}\right) /(1-q)$ when $q \neq 1$.

A useful relative of the $q$-factorial is the $q$-shifted factorial of $a$ defined by $(a ; q)_{n}=(1-a)(1-a q)\left(1-a q^{2}\right) \cdots\left(1-a q^{n-1}\right)$. The precise relationship between the two is given by $[n] !=(q ; q)_{n} /(1-q)^{n}$.

In 1843, Cauchy [3] proved for complex $|q|,|z|<1$ that

$$
\sum_{n=0}^{\infty} \frac{(a ; q)_{n} z^{n}}{(q ; q)_{n}}=\prod_{i=0}^{\infty} \frac{1-q^{i} a z}{1-q^{i} z}
$$

Replacing $a$ by $q^{a}$ and letting $q \rightarrow 1^{-}$in (2.2) gives the binomial series

$$
\sum_{n=0}^{\infty}\left(\begin{array}{c}
a+n-1 \\
n
\end{array}\right) z^{n}=(1-z)^{-a}
$$

Two $q$-exponential functions play a central role in our discourse; namely,

$$
e_{q}(z)=\sum_{n=0}^{\infty} \frac{z^{n}}{[n] !}, \quad E_{q}(z)=\sum_{n=0}^{\infty} \frac{q^{\left(\begin{array}{c}
k \\
2
\end{array}\right)} z^{n}}{[n] !} .
$$

Note that $e_{q}(z)$ contains both the geometric series $e_{0}(z)=\sum_{n=0}^{\infty} z^{n}$ and the exponential function $e_{1}(z)=e^{z}$ as special cases. For complex $|q|,|z|<1$, the $q$-exponentials satisfy the fundamental identities

$$
e_{q}(z)=\prod_{i=0}^{\infty}\left(1-q^{i}(1-q) z\right)^{-1}, \quad E_{q}(z)=\prod_{i=0}^{\infty}\left(1+q^{i}(1-q) z\right) .
$$

As $\prod_{i=0}^{\infty}\left(1-q^{i}(1-q) z\right)^{-1}=\lim _{n \rightarrow \infty} \prod_{i=0}^{n}\left(1-q^{i} z /[n]\right)^{-1}$, the first formula in (2.5) is seen to be a $q$-analog of the classic calculus limit

$$
e^{z}=\lim _{n \rightarrow \infty}\left(1-\frac{z}{n}\right)^{-n}
$$

Similarly, the second is a $q$-analog of $e^{z}=\lim _{n \rightarrow \infty}(1+z / n)^{n}$.

The formulas in (2.5) are more commonly stated in a form with $z$ replaced by $z(1-q)$ and are known as Euler's identities. Their significance in the theory of partitions is discussed in Andrews [1]. Proofs of Euler's identities and of the more general $q$-binomial series (2.2) may be found in $[1,6]$. 
The $q$-derivative of a function $f$ is defined by

$$
f^{*}(z)=\frac{f(z)-f(z q)}{(1-q) z}
$$

As expected, $\lim _{q \rightarrow 1} f^{*}=f^{\prime}$. Also, the $q$-derivative has many properties analogous to the usual derivative. It is easy to show that $\left(z^{n}\right)^{*}=[n] z^{n-1},\left(e_{q}(z)\right)^{*}=$ $e_{q}(z)$, and that $(f(z) g(z))^{*}=f^{*}(z) g(z)+f(z) g^{*}(z q)$.

3. Mahonian statistics. A statistic $s: S_{n} \rightarrow\{0,1,2, \ldots, n(n-1) / 2\}$ is said to be Mahonian if

$$
\sum_{\sigma \in S_{n}} q^{s(\sigma)}=[n] !
$$

Note that (3.1) is a $q$-analog of the fact that $\left|S_{n}\right|=n !$. As Garsia and Remmel [5] would say, (3.1) is just a $q$-counting of permutations.

The Descent set, major index, and inversion number of a permutation $\sigma \in S_{n}$ are, respectively, defined as Des $\sigma=\{i: 1 \leq i \leq n-1, \sigma(i)>\sigma(i+1)\}$,

$$
\operatorname{maj} \sigma=\sum_{i \in \operatorname{Des} \sigma} i, \quad \operatorname{inv} \sigma=|\{(i, j): 1 \leq i<j \leq n, \sigma(i)>\sigma(j)\}|
$$

for $\sigma=634512 \in S_{6}$, Des $\sigma=\{1,4\}$, maj $\sigma=1+4=5$, and inv $\sigma=11$.

Rodriguez [14] in 1839 and MacMahon [10] in 1913, respectively, showed that $\sum_{\sigma \in S_{n}} q^{\text {inv } \sigma}=[n]$ ! and $\sum_{\sigma \in S_{n}} q^{\operatorname{maj} \sigma}=[n]$ !. The adjective "Mahonian" was coined for such statistics to honor MacMahon.

Many new Mahonian statistics have been discovered since then. Mentioning but two families, Rawlings [11] noted for any integer $r, 1 \leq r \leq n$, that the statistic

$$
\operatorname{ind}_{r} \sigma=|\{(i, j): 1 \leq i<j \leq n, \sigma(i)>\sigma(j)>\sigma(i)-r\}|+\sum i
$$

where the sum is over the set $\{i: 1 \leq i \leq n-1, \sigma(i) \geq \sigma(i+1)+r\}$ is Mahonian on $S_{n}$. Note that both the major index and the inversion number are special

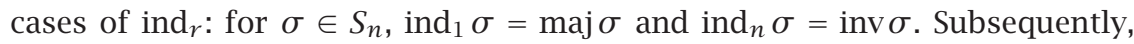
Han [9] determined a larger class that, in particular, contains Denert's statistic den, which for $\sigma \in S_{n}$, is defined to be the number of ordered pairs $(i, j)$, $1 \leq i<j \leq n$, satisfying

(a) $\sigma(i) \leq j$ or $\sigma(i)>\sigma(j)$ if $\sigma(j)>j$ or

(b) $\sigma(j) \leq \sigma(i) \leq j$ if $\sigma(j) \leq j$.

4. The Mahonian process. The Mahonian process considered in [13] consists of "Bernoulli propelling" a dot up each column of an $n \times n$ array of cells. 
In the first column, a dot is placed initially in a cell specified by a placement rule (PLR). Then, a coin that has probability $q<1$ of landing tails up is tossed until a head occurs. Each time tail is tossed, the dot moves up a cell with one exception: if tails occur when the dot is in the top cell, the dot moves to the bottom cell. When a head is tossed, the dot comes to rest. For columns 2 through $n$, the same procedure is repeated with the proviso that rows in which dots have come to rest are skipped over by subsequent dots (so, only one dot is allowed per row). For the PLR that calls for each dot to be initially placed in the lowest available cell, Example 4.1 below provides an illustration (x's have been inserted to indicate cells to be skipped over in subsequent play).

EXAMPLE 4.1.

Column 1: TH

Column 2: TTTTTH

Column 3: $\mathrm{H}$

Column 4: TTH

\begin{tabular}{|c|c|c|c|}
\hline & $\bullet$ & $\mathrm{x}$ & $\mathrm{x}$ \\
\hline & & & $\bullet$ \\
\hline$\bullet$ & $\mathrm{x}$ & $\mathrm{x}$ & $\mathrm{x}$ \\
\hline & & $\bullet$ & $\mathrm{x}$ \\
\hline
\end{tabular}

The outcome may be associated with a permutation $\sigma \in S_{n}$ in a natural way: numbering the rows from bottom to top with 1 through $n$ and the columns from left to right with 1 through $n$, let $\sigma(i)$ be the number of the row in which the $i$ th dot comes to rest. In Example 4.1, $\sigma=2413 \in S_{4}$.

EXAMPLE 4.2. As a second example, note that if the PLR calls for (a) the first dot to be placed in the lowest available cell in column 1 and (b) the $i$ th dot to be initially placed in the first available cell above the row where the $(i-1)$ st dot stopped, the same Bernoulli sequence as above generates

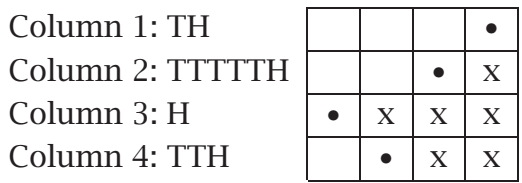

The associated permutation is $\sigma=2134 \in S_{4}$.

Relative to a PLR, the norm of a permutation $\sigma \in S_{n}$, denoted by $|\sigma|$, is defined to be the number of tails in the shortest Bernoulli sequence that generates $\sigma$. In Example 4.1, the shortest sequence that generates 2413 is THTTHHH and $|2413|=3$. In Example 4.2, $|2134|=3$.

By noting that the probability of a dot in the $i$ th column coming to rest in the $k$ th empty cell, $0 \leq k \leq n-i$, in advance of its initial placement is

$$
q^{k}(1-q)\left(1+q^{n-i+1}+q^{2(n-i+1)}+\cdots\right)=\frac{q^{k}(1-q)}{1-q^{n-i+1}}=\frac{q^{k}}{[n-i+1]},
$$

Rawlings [13] proved the following theorem. 
THEOREM 4.3. For any PLR and $0 \leq q<1$, the probability of $\sigma \in S_{n}$ being generated by the Mahonian process is

$$
M_{n, q}(\sigma)=\frac{q^{|\sigma|}}{[n] !}
$$

The measure $M_{n, q}$ is a $q$-analog of the uniform one on $S_{n}$ : $\lim _{q \rightarrow 1^{-}} M_{n, q}(\sigma)=$ $1 / n$ !. Another interesting side of Theorem 4.3 is that it supplies a whole family of Mahonian statistics. As $\sum_{\sigma \in S_{n}} M_{n, q}(\sigma)=1$, the following corollary is immediate.

COROLLARY 4.4. Relative to any PLR, the norm $|\cdot|$ is Mahonian, that is,

$$
\sum_{\sigma \in S_{n}} q^{|\sigma|}=[n] !
$$

Corollary 4.4 easily gives $n$ ! plus Mahonian statistics on $S_{n}$. To see how, note that there are $n$ different cells to initially place a dot in the first column, $n-1$ cells to initially place a dot in the second column, and so on. Thus, there are at least $n$ ! distinct PLRs. Moreover, running the process with an altered order on the columns (say from the rightmost to leftmost columns) gives rise to yet more such statistics.

As noted in [13], the family of Mahonian statistics arising in Corollary 4.4 coincides with the one discovered by Han [9]. For the Mahonian process run columnwise from left to right, Treadway and Rawlings [15] observed for the PLRs of Examples 4.1 and 4.2, respectively, that $|\sigma|=\operatorname{inv} \sigma$ and $|\sigma|=\operatorname{comaj} \sigma$, where the comajor index is defined by comaj $\sigma=\sum_{i \in \operatorname{Des} \sigma}(n-i)$.

When the Mahonian process is run columnwise from right to left and dots are propelled down, it was further noted [13] that the permutation norm is equal to the maj, ind $\mathrm{i}_{r}$, and den for the respective PLRs that initially place the first dot in the top cell of the rightmost column and the $i$ th dot in the $i$ th column from the right and the first available cell

(i) maj PLR: below the row in which the $(i-1)$ st dot rests,

(ii) $\operatorname{ind}_{r}$ PLR: below the $(r-1)$ st row above the row in which the $(i-1)$ st dot rests (if there is no $(r-1)$ st row above, then use the top row),

(iii) den PLR: on or below the diagonal (running from upper right corner to the lower left).

5. A family of $q$-derangement problems. Recall that $D_{n}=\left\{\sigma \in S_{n}: \sigma(i) \neq\right.$ $i$ for $1 \leq i \leq n\}$. Relative to a given PLR, define

$$
d_{n}(q)=\sum_{\sigma \in D_{n}} q^{|\sigma|}
$$

As $d_{n}(1)=d_{n}$, the problem of computing $d_{n}(q)$ is a $q$-derangement problem. 
To date, only a few members of this family of problems have been solved. For $|\sigma|=\operatorname{maj} \sigma$, Gessel [7] in 1981 obtained the $q$-analog

$$
d_{n}(q)=[n] ! \sum_{k=0}^{n} \frac{(-1)^{k}}{[k] !}
$$

of (1.1) as a consequence of a more general enumerative result. The asymptotic probability of a derangement being generated in this case is then seen to be

$$
\lim _{n \rightarrow \infty} \frac{d_{n}(q)}{[n] !}=\frac{1}{e_{q}(1)}=E_{q}(-1)
$$

A bijective proof of (5.2) was later given by Wachs [16].

Formula (5.2) is also the solution for the $q$-derangement problem when $|\sigma|=\operatorname{comaj} \sigma$. As is evident from the geometry of the PLRs for maj and comaj, there is a simple explanation of this fact. The reversal and complement of a permutation $\sigma=\sigma(1) \sigma(2) \cdots \sigma(n) \in S_{n}$, respectively, are

$$
\mathscr{R}(\sigma)=\sigma(n) \cdots \sigma(1), \quad \mathscr{C}(\sigma)=(n+1-\sigma(1)) \cdots(n+1-\sigma(n)) .
$$

The map $\mathscr{R} \circ \mathscr{C}: S_{n} \rightarrow S_{n}$ is a bijection such that maj $\sigma=\operatorname{comaj} \mathscr{R} \circ \mathscr{C}(\sigma)$ and such that $\sigma$ and $\mathscr{R} \circ \mathscr{C}(\sigma)$ have the same number of fixed points. Thus, $\sum_{\sigma \in D_{n}} q^{\text {maj } \sigma}=\sum_{\sigma \in D_{n}} q^{\text {comaj } \sigma}$. More generally, the bijection $\mathscr{R} \circ \mathscr{C}$ exposes the equivalence of $q$-derangement problems relative to the Mahonian process run columnwise from left to right with dots going up to those arising when the process is run columnwise from right to left with dots going down.

When $|\sigma|=\operatorname{inv} \sigma$, Griffin [8] in 1996 proved that there is a striking discontinuity in the asymptotic probability of a derangement being generated; namely,

$$
\lim _{n \rightarrow \infty} \frac{d_{n}(q)}{[n] !}= \begin{cases}\frac{1}{e} & \text { if } q=1 \\ 0 & \text { if } 0 \leq q<1\end{cases}
$$

He gave no closed formula for $d_{n}(q)$.

At first glance, the $q$-derangement problems solved by Garsia and Remmel [5] and by Rawlings [11] do not appear to fit into the framework of the Mahonian process.

6. Cycle placement rules. From a probabilistic point of view, the primary difficulty in solving a $q$-derangement problem relative to the Mahonian process lies in the fact that the generation of fixed points is not columnwise independent. Beyond a few conjectures and heuristic explanations in Section 7, we 
have little new to say in this regard. However, by adding a twist to the process of Section 4 , we discovered a class of $q$-derangement problems for which an appeal to independence may be made.

The twist consists of letting the process more or less determine the column order as it runs. We begin by Bernoulli propelling a dot up the first (leftmost) column. If the dot stops in the $i$ th row (from the bottom) with $i \neq 1$, then the second dot is propelled up the $i$ th column. If the second dot comes to rest in the $j$ th row with $j \neq 1$, then the third dot is propelled up the $j$ th column. This is continued until a dot comes to rest in the first row. The procedure is repeated until no empty column remains. For instance, if each dot is initially inserted in the second lowest available cell (with the exception that the last dot is inserted in the only available cell), then the Bernoulli sequence TTHHTTHHH generates the outcome in the following example.

EXAMPLE 6.1.

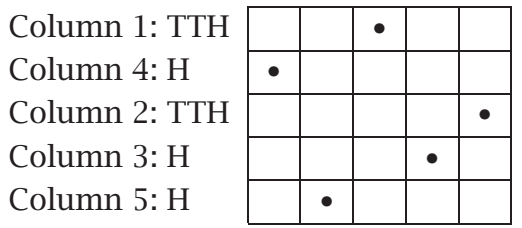

The associated permutation is $\sigma=41523 \in S_{5}$.

Note that if $\sigma=c_{1} c_{2} \cdots c_{k}$, where $c_{1}, c_{2}, \ldots, c_{k}$ are disjoint cycles such that the minimum element in each $c_{j}$ appears at the left in $c_{j}$ and the minimum of $c_{j-1}$ is less than the minimum of $c_{j}$ for $2 \leq j \leq k$, then the column order corresponds with the order of the integers in $c_{1} c_{2} \cdots c_{k}$ read from left to right. In Example 6.1, $\sigma=(142)$ (35) and the column order is 1,4,2,3,5. Thus, the column order is compatible with the cycle structure of the associated permutation.

We use the term cycle placement rule (CPLR) when the columns are to be selected as in Example 6.1. It should be noted that Theorem 4.3 and its corollary remain valid for CPLRs.

7. Four CPLR q-derangement problems. Under the CPLR of Example 6.1 that calls for each dot to be placed in the second lowest available cell, a derangement is generated precisely when 1 winds up in a $k$-cycle, $2 \leq k \leq n$ and the remaining $n-k$ elements are deranged. As knowledge of the first cycle in no way influences the generation of subsequent cycles, it follows that the probability of a derangement being generated satisfies the recurrence

$$
\frac{d_{n}(q)}{[n] !}=\sum_{k=2}^{n} \operatorname{Prob}\left(1 \text { is in a } k \text {-cycle) } \frac{d_{n-k}(q)}{[n-k] !}\right.
$$


for $n \geq 2$. In view of (4.1), the probability that 1 is in a 1 -cycle is $q^{n-1} /[n]$. So, 1 is not in a 1 -cycle with probability $1-q^{n-1} /[n]=[n-1] /[n]$. Repeated use of (4.1) then leads to

$$
\operatorname{Prob}(1 \text { is in a } k \text {-cycle })=\frac{q^{n-k}}{[n-k]} \prod_{i=1}^{k-1} \frac{[n-i]}{[n-i+1]}=\frac{q^{n-k}}{[n]} \text {. }
$$

Thus,

$$
\frac{d_{n}(q)}{[n-1] !}=q^{n} \sum_{k=2}^{n} q^{-k} \frac{d_{n-k}(q)}{[n-k] !}
$$

for $n \geq 2$. The appropriate initial conditions are $d_{0}(q)=1$ and $d_{1}(q)=0$.

The power of generating functions may now be brought to bear. Define

$$
D(z)=\sum_{n \geq 0}^{\infty} \frac{d_{n}(q)}{[n] !} z^{n}
$$

As $0 \leq d_{n}(q) /[n] ! \leq 1, D(z)$ certainly converges in the complex disk $|z|<1$.

Multiplying both sides of (7.3) by $z^{n}$ and then summing over $n \geq 2$ yields the $q$-differential equation

$$
D^{*}(z)=\frac{z}{1-z} D(z q)
$$

with the initial condition $D(0)=1$. Letting $q \rightarrow 1^{-}$in (7.5) results in the separable equation $D^{\prime}=z D /(1-z)$, which, when solved, gives the well-known exponential generating function for the derangement numbers; namely,

$$
\sum_{n \geq 0}^{\infty} \frac{d_{n}}{n !} z^{n}=\frac{e^{-z}}{1-z}
$$

So, how does one solve the $q$-differential equation in (7.5) for $0 \leq q<1$ ? The solution is actually as simple as the case $q=1$. Using the definition of the $q$-derivative and a little algebra, (7.5) may be rewritten as

$$
D(z)=\frac{(1-z / \alpha)(1-z / \beta)}{1-z} D(z q)
$$

where

$$
\alpha=\frac{1+\sqrt{4 q-3}}{2(1-q)}, \quad \beta=\frac{1-\sqrt{4 q-3}}{2(1-q)} .
$$


Iteration of (7.7) then leads to

$$
D(z)=D\left(z q^{n+1}\right) \prod_{i=0}^{n} \frac{\left(1-q^{i} z / \alpha\right)\left(1-q^{i} z / \beta\right)}{1-q^{i} z}
$$

As $\sum_{i=0}^{\infty} q^{i}$ converges absolutely for $|q|<1$, the products (see [2, page 208]) $\prod_{i=0}^{\infty}\left(1-q^{i} z / \alpha\right), \prod_{i=0}^{\infty}\left(1-q^{i} z / \beta\right)$, and $\prod_{i=0}^{\infty}\left(1-q^{i} z\right)$ are all absolutely convergent (to nonzero values) in some open disk containing the origin. As $D$ is continuous at the origin, taking the limit as $n \rightarrow \infty$ in (7.9) therefore gives

$$
D(z)=\prod_{i=0}^{\infty} \frac{\left(1-q^{i} z / \alpha\right)\left(1-q^{i} z / \beta\right)}{1-q^{i} z} .
$$

Towards writing (7.10) in a form analogous to (7.6), note that (2.5) implies

$$
D(z)=\frac{E_{q}(-2 z /(1+\sqrt{4 q-3}))}{1-z} \prod_{i=0}^{\infty} \frac{1-q^{i} z / \beta}{1-q^{i+1} z} .
$$

Then, the fact that $\lim _{q \rightarrow 1^{-}} \prod_{i=0}^{\infty}\left(1-q^{i} z / \beta\right) /\left(1-q^{i+1} z\right)=1$ (left as an exercise) shows that (7.6) is indeed the limit of (7.10) as $q \rightarrow 1^{-}$.

With the aid of (2.2), we may further extract a closed formula for our $q$ derangement problem from (7.11); namely,

$$
\frac{d_{n}(q)}{[n] !}=\sum_{k=0}^{n} \frac{q^{\left(\begin{array}{c}
k \\
2
\end{array}\right)(-2)^{k}(1 / \beta ; q)_{n-k}}}{[k] !(1+\sqrt{4 q-3})^{k}(q ; q)_{n-k}} .
$$

To compute the asymptotic probability, it is tempting to apply the analytic fact that if $\left\{a_{n}\right\}$ converges to $a$, then $a=\lim _{z \rightarrow 1^{-}}(1-z) \sum_{n=0}^{\infty} a_{n} z^{n}$ directly to (7.10). However, we do not know a priori that $\lim _{n \rightarrow \infty} d_{n}(q) /[n]$ ! exists. So, we take a more cautious approach.

First note that if a series $\sum_{k=0}^{\infty} A_{k}=A$ of complex numbers converges absolutely and a complex sequence $\left\{B_{n}\right\}$ converges to $B$, then

$$
\lim _{n \rightarrow \infty} \sum_{k=0}^{n} A_{k} B_{n-k}=A B
$$

An application of the ratio test reveals that $\sum_{k=0}^{n} q^{\left(\begin{array}{c}k \\ 2\end{array}\right)}(-2)^{k} /[k] !(1+\sqrt{4 q-3})^{k}$ converges absolutely for $|q|<1$. Also, $\lim _{n \rightarrow \infty}(1 / \beta ; q)_{n}=\prod_{i=0}^{\infty}\left(1-q^{i} / \beta\right)$ and $\lim _{n \rightarrow \infty}(q ; q)_{n}=\prod_{i=0}^{\infty}\left(1-q^{i}\right)$ converge absolutely (to nonzero values). So, 
TABLE 7.1

\begin{tabular}{|l|c|c|}
\hline Placement & $\alpha, \beta$ & $D(z)$ \\
\hline CPLR1 & $\frac{1 \pm \sqrt{4 q-3}}{2(1-q)}$ & $\frac{E_{q}(-2 z /(1+\sqrt{4 q-3}))}{1-z} \prod_{i=0}^{\infty} \frac{1-q^{i} z / \beta}{1-q^{i+1} z}$ \\
\hline CPLR2 & $\frac{-q \pm \sqrt{4 q-3 q^{2}}}{2 q(1-q)}$ & $\frac{e_{q}\left(-2 q z /\left(q+\sqrt{4 q-3 q^{2}}\right)\right)}{1-z} \prod_{i=0}^{\infty} \frac{1-z q^{i}}{1-q^{i} z / \alpha}$ \\
\hline CPLR3 & $\frac{1 \pm \sqrt{1-4 q(1-q)}}{2 q(1-q)}$ & $\frac{E_{q}(-2 q z /(1+\sqrt{1-4 q(1-q)})}{1-z} \prod_{i=0}^{\infty} \frac{1-q^{i} z / \beta}{1-q^{i+1} z}$ \\
\hline CPLR4 & $\frac{-q \pm \sqrt{q^{2}+4(1-q)}}{2(1-q)}$ & $\frac{e_{q}\left(-2 z /\left(q+\sqrt{q^{2}+4(1-q)}\right)\right.}{1-z} \prod_{i=0}^{\infty} \frac{1-q^{i} z}{1-q^{i} z / \alpha}$ \\
\hline
\end{tabular}

(7.12) and (7.13) imply

$$
\lim _{n \rightarrow \infty} \frac{d_{n}(q)}{[n] !}=E_{q}\left(\frac{-2}{1+\sqrt{4 q-3}}\right) \prod_{i=0}^{\infty} \frac{1-q^{i} / \beta}{1-q^{i}} .
$$

The above approaches (1.2) as $q \rightarrow 1^{-}$.

There are three other CPLRs for which the associated $q$-derangement problem may be solved in much the same way as the above one. Without going into detail, we record the respective generating functions in Table 7.1 for the CPLRs calling for

(i) CPLR1: each dot to be placed in the second lowest available cell,

(ii) CPLR2: each dot to be placed in the lowest available cell,

(iii) CPLR3: each dot associated with the beginning of a new cycle to be placed in the lowest available cell, while all other dots are placed in the second lowest available cell,

(iv) CPLR4: each dot associated with the beginning of a new cycle to be placed in the second lowest available cell, while all other dots are placed in the lowest available cell.

We note that the asymptotic probability of a derangement being generated under CPLR2 and CPLR4 exhibits the same discontinuity as in (5.5).

The generating functions for CPLR1 and CPLR2 coincide exactly with ones derived earlier in [12] that count permutations having no minimum components by inversion number. Our treatment here is entirely different.

8. Some heuristic arguments and conjectures. We restrict our attention to the Mahonian process run as initially described, that is, with the column order being from left to right. Our comments in this section are far less than rigorous. 
Relative to the comaj-PLR of Example 4.2, Griffin gave a very believable heuristic explanation (unpublished) for why

$$
\lim _{n \rightarrow \infty} \frac{d_{n}(q)}{[n] !}=E_{q}(-1)
$$

based on the product expansion

$$
E_{q}(-1)=\prod_{i=0}^{\infty}\left(1-q^{i}(1-q)\right) .
$$

As the process runs, the trajectory of the dots winds itself around the $n \times n$ array. On the $(i+1)$ st upswing through the array, the process crosses the diagonal for the $(i+1)$ st time (except possibly on the last upswing). The probability that a fixed point occurs say in row $k$ on the $(i+1)$ st upswing is equal to the probability that no dot stopped in row $k$ on the $i$ previous upswings (which is $\left.q^{i}\right)$ times the probability that a dot on the $(i+1)$ st upswing comes to rest in row $k$ (which is $(1-q)$ ). Thus, if $\omega(n)$ denotes the expected number of upswings, then the approximate probability of a derangement in $S_{n}$ being generated is

$$
\prod_{i=0}^{\omega(n)}\left(1-q^{i}(1-q)\right) .
$$

As $\lim _{n \rightarrow \infty} \omega(n)=\infty,(8.1)$ is seen to indeed be plausible. The gap in the argument lies in proving that the error made in (8.3) goes to 0.

Griffin's heuristic viewpoint may be modified to give a few conjectures regarding other PLRs. Let $\rho_{i}$ denote the number of dots that enter row $i$ in the first $i-1$ columns. We conjecture that a PLR for which both

(i) the expected value of $\rho_{i}$ and

(ii) the number of tails required for the $i$ th dot to reach the $i$ th row from its initial placement

are uniformly bounded by $M$ will generate a derangement with asymptotic probability 0 for $q<1$. Under such a PLR, the probability of a fixed point in the $i$ th column is expected to be less than or equal to $c=\left(1-q^{2 M}(1-q)\right)$. As $c<1$, a product involving infinitely many such factors diverges to 0 . From Griffin's work [8], it may be shown that the inv-PLR of Example 4.1 falls into this category. We feel that the den-PLR is also essentially of this type.

Based on limited numerical work, we further conjecture that the PLRs calling for the $i$ th dot to be placed in

(i) the first available cell above the diagonal (from the lower left corner to the upper right),

(ii) the second available cell above the row in which the $(i-1)$ st dot rests (with the first dot being initially placed in row 1 )

generate, for $q<1$, derangements with the respective asymptotic probabilities of 1 and $q / E_{q}(1)$. 
ACKNOWLEDGMENT. This work is based on a work supported by the National Science Foundation (NSF) under Grant 0097392.

\section{REFERENCES}

[1] G. E. Andrews, The Theory of Partitions, Encyclopedia of Mathematics and Its Applications, vol. 2, Addison-Wesley Publishing, Massachusetts, 1976.

[2] T. M. Apostol, Mathematical Analysis, 2nd ed., Addison-Wesley Publishing, Massachusetts, 1974.

[3] A.-L. Cauchy, Mémoire sur les fonctions dont plusieurs valeurs sont liées entre elles par une équation linéaire, et sur diverses transformations de produits composés d'un nombre indéfini de facteurs, C. R. Acad. Sci. Paris 17 (1893), 523 (French).

[4] P. R. de Montmort, Essay d'Analyse sur les Jeux de Hazard [Essay on the Analysis of Games of Chance], Chelsea Publishing, New York, 1980 (French), reprint of the 1708 edition.

[5] A. M. Garsia and J. Remmel, A combinatorial interpretation of q-derangement and q-Laguerre numbers, European J. Combin. 1 (1980), no. 1, 47-59.

[6] G. Gasper and M. Rahman, Basic Hypergeometric Series, Encyclopedia of Mathematics and Its Applications, vol. 35, Cambridge University Press, Cambridge, 1990.

[7] I. Gessel, Counting permutations by descents, greater index, and cycle structure, MIT, preprint, 1981.

[8] K. Griffin, The q-derangement problem relative to the inversion number, senior project, California Polytechnic State University, California, 1996.

[9] G.-N. Han, Une transformation fondamentale sur les réarrangements des mots [A fundamental transformation on rearrangements of words], Adv. Math. 105 (1994), no. 1, 26-41 (French).

[10] P. A. MacMahon, The indices of permutations and the derivation therefrom of functions of a single variable associated with the permutations of any assemblage of objects, Amer. J. Math. 35 (1913), 281-322.

[11] D. Rawlings, The r-major index, J. Combin. Theory Ser. A 31 (1981), no. 2, 175183.

[12] _ The ABC's of classical enumeration, Ann. Sci. Math. Québec 10 (1986), no. 2, 207-235.

[13] _ A generalized Mahonian statistic on absorption ring mappings, J. Combin. Theory Ser. A 79 (1997), no. 2, 255-267.

[14] O. Rodriguez, Note sur les inversions, ou dérangements produits dans les permutations, J. de Math. 4 (1839), 236-240 (French).

[15] J. Treadway and D. Rawlings, Bernoulli trials and Mahonian statistics: a tale of two q's, Math. Mag. 67 (1994), no. 5, 345-354.

[16] M. L. Wachs, On q-derangement numbers, Proc. Amer. Math. Soc. 106 (1989), no. $1,273-278$.

Jessica Delfert, Hillary Einziger, and Don Rawlings: Mathematics Department, California Polytechnic State University, San Luis Obispo, CA 93407, USA 


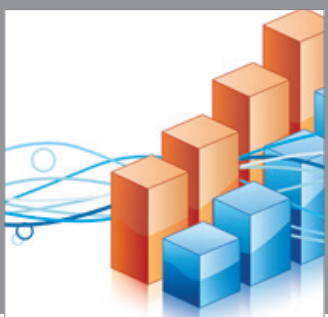

Advances in

Operations Research

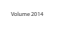

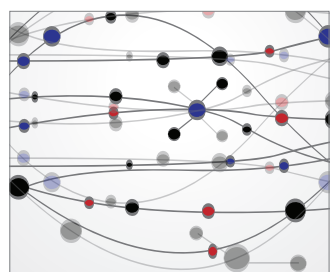

\section{The Scientific} World Journal
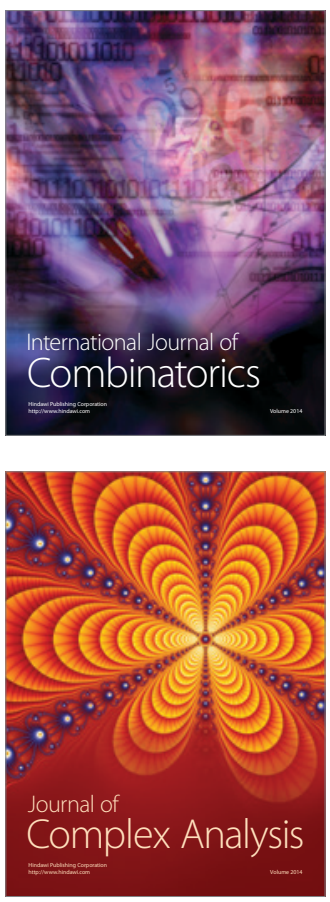

International Journal of

Mathematics and

Mathematical

Sciences
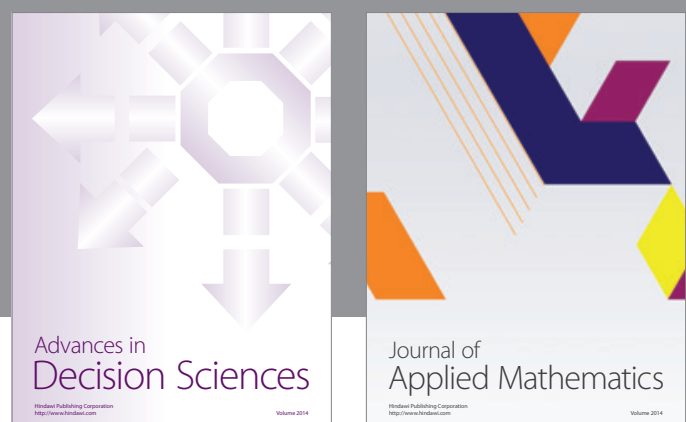

Journal of

Applied Mathematics
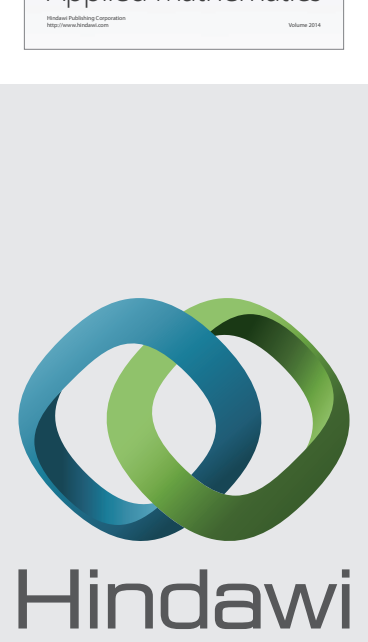

Submit your manuscripts at http://www.hindawi.com
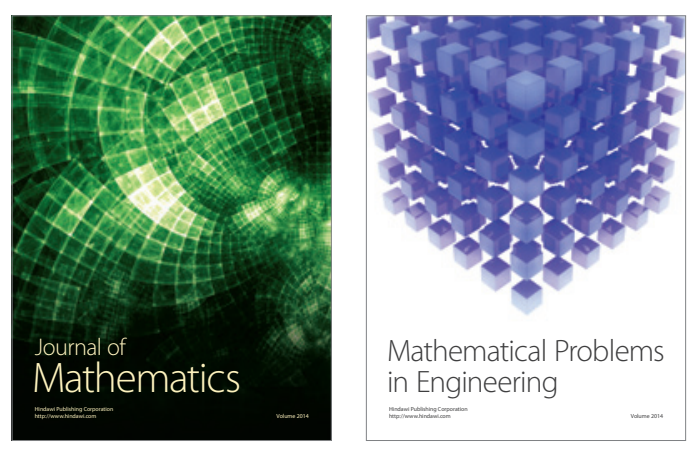

Mathematical Problems in Engineering
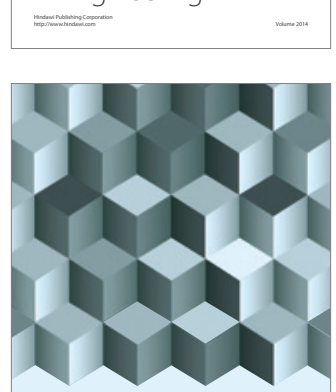

Journal of

Function Spaces
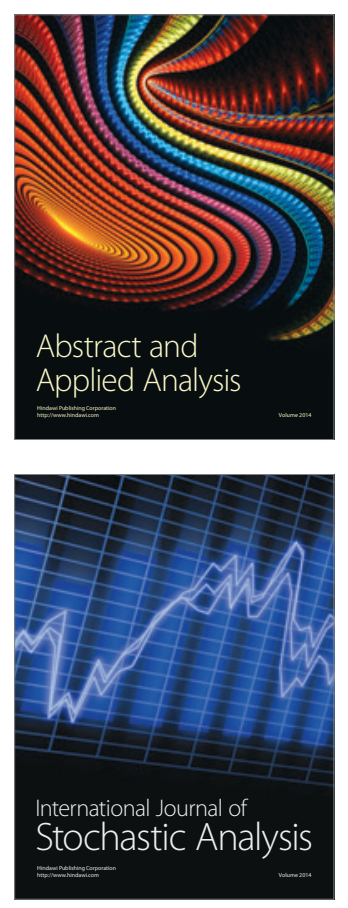

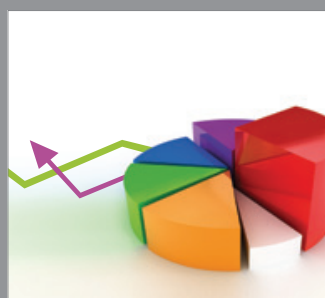

ournal of

Probability and Statistics

Promensencen
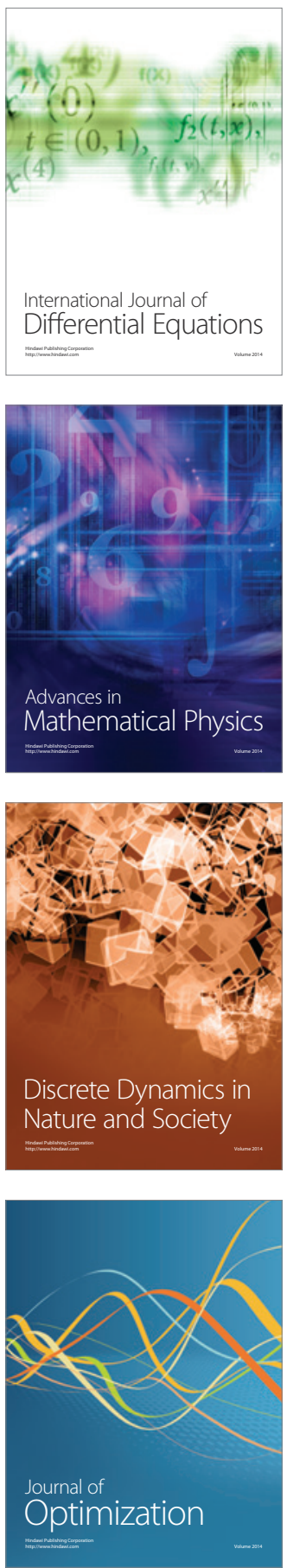